\title{
LAS FORMACIONES DE RUDISTAS DE LA PLATAFORMA DE SANT CORNELI (CRETÁCICO SUPERIOR, UNIDAD CENTRAL SURPIRENAICA)
}

\author{
Eulalia GILI' ${ }^{1}$ Enric VICENS ${ }^{1}$, Antonio OBRADOR ${ }^{1}$ Peter W. SKELTON ${ }^{2}$ \\ y Gregorio LÓPEZ ${ }^{1}$
}

' Departament de Geologia. Universitat Autònoma de Barcelona, Bellaterra 08193.

${ }^{2}$ Department of Earth Sciences. The Open University, Milton Keynes, MK7 6A A, G.B.

Gili, E., Vicens, E., Obrador, A., Skelton, P.W. y López, G. 1996. Las formaciones de rudistas de la plataforma de Sant Corneli (Cretácico Superior, unidad central surpirenaica). [The rudist formations of the Sant Corneli platform (Upper Cretaceous, South Pyrenean Central Unit]. Revista Española de Paleontología, Nºxtraordinario, 172-181. ISSN 0213-6937.

\begin{abstract}
Upper Cretaceous carbonate platform facies are present in both limbs of the E-W oriented Sant Corneli Anticline in the southern Central Pyrenees. We have made a detailed palaeoenvironmental analysis of a rich rudist and coral formation of Santonian age, in its northern limb.

The platform succession crops out for at least $6,8 \mathrm{Km} \mathrm{E-W}$ and $1,25 \mathrm{Km} \mathrm{N}-\mathrm{S}$. In its western part, miliolid-rich quartzose calcarenites were transported in from the SW. The rudist and coral formation developed mainly in its outer, northeastern part. A repeated assemblage succession in the latter begins with a coral-dominated assemblage, and passes up via a mixed coral and rudist assemblage to a paucispecific hippuritid lithosome. The lower units of such successions represent relatively more open, normal marine conditions, and the uppermost hippuritid units more restricted marine conditions. This assemblage succession was formed in response to shallowing caused by sediment accumulation.

Detailed study of one of the hippuritid lithosomes shows that the hippuritid elevator congregations grew constratally (i.e. without any supporting biogenic framework), and that they lacked relief. So these rudist grew as gregarious sediment-dwellers, not reef-builders, in the restricted waters of the platform top. Here they were sporadically disturbed by storms, and eventually blanketed by bioclastic material swept in from the platform margin.
\end{abstract}

Keywords: Rudist bivalves, Calcareous platform, Stratigraphy, Palaeoecology, Upper Cretaceous (Santonian), Pyrenees.

\section{RESUMEN}

En ambos flancos del anticlinal de Sant Corneli, en la unidad central surpirenaica, afloran facies de plataforma calcárea de edad Santoniense. En este trabajo, hemos realizado un análisis paleoambiental detallado de la formación de rudistas y corales del flanco norte.

La sección estudiada tiene una extensión de $6,8 \mathrm{Km}$ de este a oeste y de 1,25 $\mathrm{Km}$ de norte a sur. En el oeste hay calcarenitas cuarzosas, ricas en miliólidos, transportadas desde el sudoeste. En el nordeste, en su parte más externa, se desarrolló una formación de rudistas y corales. Los ciclos que forman dicha formación empiezan con unas margas con abundantes corales en la base y asociaciones mixtas de corales y rudistas hacia arriba, que pasan a un tramo calcáreo constituido por agregados de hipurítidos. Los tramos inferiores de estas sucesiones representan condiciones marinas normales, relativamente más abiertas, y los litosomas de hipurítidos de la parte superior condiciones marinas más restringidas. Esta sucesión faunística se formó en respuesta a la somerización causada por la acumulación de sedimento.

Del estudio detallado de uno de los litosomas de hipurítidos, se concluye que los agregados de hipurítidos carecían de estructura orgánica rígida y relieve original. Estos rudistas, por lo tanto, crecieron como pobladores de sedimentos, no como constructores arrecifales, en aguas restringidas de la parte superior de la plataforma. Allí, ocasionalmente eran perturbados por tempestades, y finalmente cubiertos por sedimentos bioclásticos transportados del margen de la plataforma.

Palabras clave: Rudistas (Bivalvia), Plataforma calcárea, Estratigrafía, Paleoecología, Cretácico superior (Santoniense), Pirineos.

\section{INTRODUCCIÓN}

La unidad central surpirenaica (Seguret, 1972) está caracterizada por una serie de láminas cabalgantes, imbricadas y desplazadas hacia el sur originadas entre el Cretácico superior y el Mioceno inferior, como consecuencia de la convergencia entre las placas ibérica y europea (Muñoz, 1992).
Una de estas unidades corresponde al manto de Boixols, en cuyo frente meridional se sitúa el anticlinal de Sant Corneli (Fig. 1). En ambos flancos de este anticlinal afloran sedimentos de plataforma carbonatada con importantes formaciones de rudistas.

Estudios de detalle en el flanco sur, les Collades de Basturs, condujeron al establecimiento de un modelo de pla- 


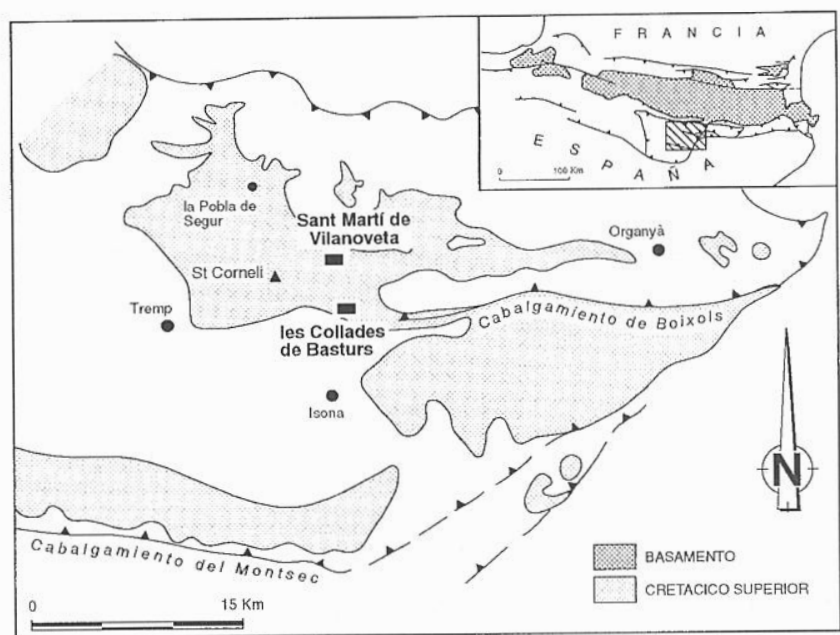

Figura 1. Situación de las formaciones de rudistas de St. Corneli (Sant Martí de Vilanoveta y les Collades de Basturs) en un esquema estructural simplificado de los Pirineos.

taforma caracterizada por presentar, de este a oeste, una zona interna de circulación marina restringida con construcciones de rudistas y una zona externa, más abierta, con construcciones mixtas de rudistas y corales que pasa gradualmente a sedimentos de talud superior (Gili, 1984, 1992, 1993). Siguiendo esta misma línea de investigación, centrada en el estudio de la paleoecología de las formaciones de rudistas, hemos analizado el área norte de la sierra de Sant Corneli, sección de Sant Martí de Vilanoveta, estudiada anteriormente por Ross (1989) y hemos establecido, así mismo, un modelo para el desarrollo de los litosomas de rudistas de esta sección.

Los trabajos publicados hasta la fecha por otros autores sobre las formaciones de rudistas y corales del área de Sant Corneli, llegan a conclusiones claramente diferentes por lo que se refiere a su edad. (Gili et al., 1994). Así, por un lado, Rosell (1967) considera que las formaciones de rudistas y corales de Sant Martí de Vilanoveta (Vilanoveta de Aramunt) y de les Collades de Basturs son coetáneas y de edad Santoniense medio. Mientras que, por otro lado, existe la hipótesis de que dichas formaciones son de distinta edad (Coniaciense-Santoniense inferior, Sant Martí de Vilanoveta, y Santoniense medio superior, les Collades de Basturs), y se las emplaza en unidades litoestratigráficas y secuencias deposicionales diferentes. Esta última suposición aparece en numerosos trabajos recientes, entre los que cabe destacar los de Gallemí et al. (1982 y 1983), Martínez (1982) y Simó (1993). Así pues, la principal divergencia está en la edad de la formación de rudistas y corales de Sant Martí de Vilanoveta que, en la literatura geológica de esta región, ha oscilado desde el Coniaciense hasta el Santoniense.

Además, cabe destacar que muchos de los trabajos publicados sobre esta área, especialmente los referentes a estudios de estratigrafía secuencial, carecen de un control bioestratigráfico preciso, o, en cualquier caso, adolecen de falta de datos bioestratigráficos que justifiquen su interpretación.

Así, la problemática expuesta anteriormente, en cuanto a la datación de estas formaciones de rudistas y corales, hacía necesario y obligatorio un estudio bioestratigráfico detallado, en especial de la sección de Sant Martí de Vilanoveta.

Los objetivos del presente trabajo son, por una parte, la datación, descripción e interpretación deposicional de la sección de Sant Martí de Vilanoveta y, por otra, el estudio detallado e interpretación paleoecológica de los litosomas de rudistas de dicha sección.

\section{ANÁLISIS BIOESTRATIGRÁFICO}

El estudio bioestratigráfico de la sección de Sant Martí de Vilanoveta, apoyado en la correlación de las unidades sedimentarias, ha proporcionado suficientes datos para concluir que su edad es Santoniense (Gili et al., 1994). De los numerosos grupos fósiles reconocidos en esta área se seleccionaron los más adecuados para el estudio bioestratigráfico y la correlación con la sección de les Collades de Basturs. En concreto, se ha estudiado detalladamente la distribución estratigráfica de los inocerámidos, ammonites, foraminíferos planctónicos y rudistas.

El estudio bioestratigráfico de la fauna de inocerámidos de la sección de Sant Martí de Vilanoveta ha permitido reconocer tres asociaciones, todas ellas pertenecientes al Santoniense. La asociación correspondiente al Santoniense inferior se reconoce por la presencia de Inoceramus (Platyceramus) undulatoplicatus michaeli Heinz, I. (Pl.) ezoensis Yokoyama, I. (Pl.) cycloides cycloides Wegner, I. (Pl.) cycloides ahsenensis Seitz e $I$. (Pl.) adversus Riedel; y sería equivalente a la Asociación con Inoceramus (Platyceramus) undulatoplicatus de López (1992), a la Zona de Undulatoplicatus de Seitz (1961) y a la Zona 26 de Tröger (1989). La asociación correspondiente al Santoniense medio se reconoce por la presencia de Inoceramus (Cordiceramus) bueltenensis bueltenensis Seitz, I. (Co.) bueltenensis arnoldi Seitz e I. (Platyceramus) cycloides cycloides Wegner. La asociación correspondiente al Santoniense superior se reconoce por la presencia de Inoceramus (Cordiceramus) brancoiformis Seitz e I. (Platyceramus) cycloides cycloides Wegner; y sería equivalente a la Asociación con Inoceramus (Platyceramus) brancoiformis de López (1992).

La determinación sistemática de la fauna de ammonites recolectada hasta la fecha, llevada a cabo por el Dr. W. J. Kennedy de la Universidad de Oxford (Gran Bretaña), ha permitido confirmar la atribución de esta sección estratigráfica al Santoniense. La mayor parte de las especies indican claramente una edad Santoniense, especialmente Texanites gallicus Collignon, Texanites sp., Pseudoschloenbachia De Grossouvre y Placenticeras polyopsis Dujardin, y corresponderían a la Zona de Placenticeras polyopsis. De todas formas, cabe mencionar que Nowakites sp. también ha sido citada en el Coniaciense de otras regiones. En la mayor parte de los trabajos sobre la bioestratigrafía de los ammonites del Santoniense se reconoce una única zona para este piso, tal es el caso del trabajo de Kennedy (1987) en el área tipo y del de Santamaría (1992) en el norte de España. Sin embargo, recientemente, Hancock (1991) subdividió el Santoniense del área tipo en dos zonas: la de Texanites gallicus (inferior) y la de Placenticeras paraplanum (superior). De todas formas, a efectos del presente trabajo se sigue aceptando como válida la Zona de Placenticeras polyopsis. Cosa muy distinta es lo 


\begin{tabular}{|c|c|c|}
\hline & S.M.V. & C.B. \\
\hline Hipuritella maestrei (Vidal) & • & • \\
\hline Hippuritella sulcatissima (Douvillé) & - & \\
\hline Hippuritella toucasi (d'Orbigny) & & - \\
\hline Hippurites canaliculatus Rolland du Roquand & $\bullet$ & \\
\hline Hippurites matheroni Douvillé & & - \\
\hline Hippurites microstylus Douvillé & - & - \\
\hline Hippurites praecessor Douvillé & - & • \\
\hline Hippurites socialis Douvillé & - & • \\
\hline Hippurites sublaevis Matheron & - & - \\
\hline Vaccinites beaussetensis Toucas & - & - \\
\hline Vaccinites chaperi (Douvillé) & & - \\
\hline Vaccinites galloprovincialis (Matheron) & $\bullet$ & - \\
\hline Vaccinites galloprovincialis ? & - & \\
\hline Vaccinites giganteus major Toucas & - & $\bullet$ \\
\hline Vaccinites zurcheri (Douvillé) & & - \\
\hline Biradiolites acuticostatus (d'Orbigny) & - & • \\
\hline Biradiolites angulosissimus Toucas & $\bullet$ & - \\
\hline Biradiolites beaussetensis Toucas & & $\bullet$ \\
\hline Biradiolites carezi Toucas & & - \\
\hline Biradiolites fissicostatus d'Orbigny & & - \\
\hline Bournonia excavata (d'Orbigny) & - & $\bullet$ \\
\hline Praeradiolites caderensis Toucas & - & - \\
\hline Praeradiolites sarladensis? Toucas & - & \\
\hline Praeradiolites plicatus (Lajard-Negrel-Toulouzan) & & - \\
\hline Radiolites galloprovincialis Matheron & - & - \\
\hline Radiolites vallispetrosae Astre & - & \\
\hline Radiolites squamosus d'Orbigny & & • \\
\hline Sauvagesia aliciae Pons & & - \\
\hline Sauvagesia tenuicostata Polsak & & - \\
\hline Bayleia pouechi Munier-Chalmas & & $\bullet$ \\
\hline Bayleia sp. & - & \\
\hline Monopleura minuta Vidal & & - \\
\hline Caprotinidae indet. & $\bullet$ & \\
\hline Plagioptychus paradoxus Matheron & - & - \\
\hline Plagioptychus toucasi Matheron & & • \\
\hline
\end{tabular}

Tabla 1. Listado de especies de rudistas de St. Martí de Vilanoveta (S.M.V.) y de les Collades de Basturs (C.B.); éste último según Gili (1993), basado en Pons (1974), Gili (1984) y Gili y Pons (1988).

que sucede con los inocerámidos, que sí han permitido realizar subdivisiones válidas para el Santoniense de muchas regiones.

Por su parte, el estudio preliminar de la fauna de foraminíferos planctónicos, realizada por el Dr. E. A. M. Koutsoukus de Petrobras (Brasil), ratifica la edad de Santoniense para la sección de Sant Martí de Vilanoveta. Concretamente, el análisis de algunas muestras con abundantes foraminíferos planctónicos de los tramos margosos, de la unidad de margas y arcillas (Fig. 2), ha permitido reconocer la Biozona de Dicarinella asymetrica, que corresponde al Santoniense superior. Pormenorizando, las especies de foraminíferos planctónicos reconocidas en esta biozona son: Dicarinella asymetrica (Sigal), Dicarinella concavata (Brotzen), Margi- notruncana pseudolinneiana Pessagno, Marginotruncana $\mathrm{cf}$. schneegansi (Sigal) y Globotruncana bulloides Vogler.

Aunque el estudio detallado de la fauna de rudistas y su repartición estratigráfica, no ha permitido llevar a cabo estudios bioestratigráficos detallados, sí que ha permitido poner de manifiesto la semejanza entre la fauna de rudistas de Sant Martí de Vilanoveta y de les Collades de Basturs (Tab. 1). De hecho, la principal diferencia observada entre la fauna de rudistas de ambas áreas radica en que se ha reconocido una mayor presencia de radiolítidos en les Collades de Basturs que en Sant Martí de Vilanoveta.

En resumen, el estudio bioestratigráfico realizado en la sección de Sant Martí de Vilanoveta permite atribuir una edad Santoniense a la unidad de rudistas y corales de dicha sección; en concreto, la citada unidad se situaría en el intérvalo comprendido entre la parte superior del Santoniense inferior y la parte media del Santoniense superior. Por lo que respecta al estudio bioestratigráfico de les Collades de Basturs, aunque si bien su fauna debe ser revisada de forma más exhaustiva, todos los datos de que disponemos no implican ninguna modificación sustancial de la edad (Santoniense) que se le ha venido atribuyendo en la literatura (Gili, 1993).

\section{ANÁLISIS LITOESTRATIGRÁFICO}

El análisis estratigráfico de la sección de Sant Martí de Vilanoveta se ha realizado mediante el levantamiento capa a capa de 13 series correlacionables lateralmente de oeste a este a lo largo de $6,8 \mathrm{Km}$ del valle del rio Carreu, entre las vecindades de los pueblos abandonados de Aramunt vell en el este y Sant Martí de Vilanoveta en el oeste. Un esquema sinóptico de la estratigrafía de una parte de dicha sección se muestra en la Fig. 2.

En los alrededores del pueblo abandonado de Aramunt vell, al oeste de la sección representada en la Fig. 2, hay una sucesión continua de calcarenitas ocres, conocida como Miembro Aramunt vell (Gallemí et al., 1982). Hacia el este, esta unidad se presenta dividida en dos partes por una cuña (Fig. 2) formada por margas con abundantes corales e intercalaciones de calizas margosas, y calizas de rudistas (unidad de rudistas y corales) en la parte inferior, y arcillas con intercalaciones de calizas margosas (unidad de margas y arcillas) en la mitad superior. En el techo de las calcarenitas ocres superiores, se observa un fondo endurecido sobre el que yacen las arcillas margosas del Miembro arcillas de Herbasavina (Gallemí et al., 1982).

En términos generales, la sección representa una plataforma carbonatada constituida por calcarenitas procedentes del sudoeste (Miembro Aramunt vell) y facies de rudistas y corales (unidad de rudistas y corales) formadas en el nordeste, en la zona externa de la plataforma.

\section{DESCRIPCIÓN DE LAS UNIDADES LITOLÓGICAS}

\section{Miembro calcarenitas de Aramunt vell (parte inferior)}

Esta parte de la sección está formada principalmente por una sucesión de ciclos granocrecientes con estratificación oblicua unidireccional. Cada ciclo empieza con calizas 


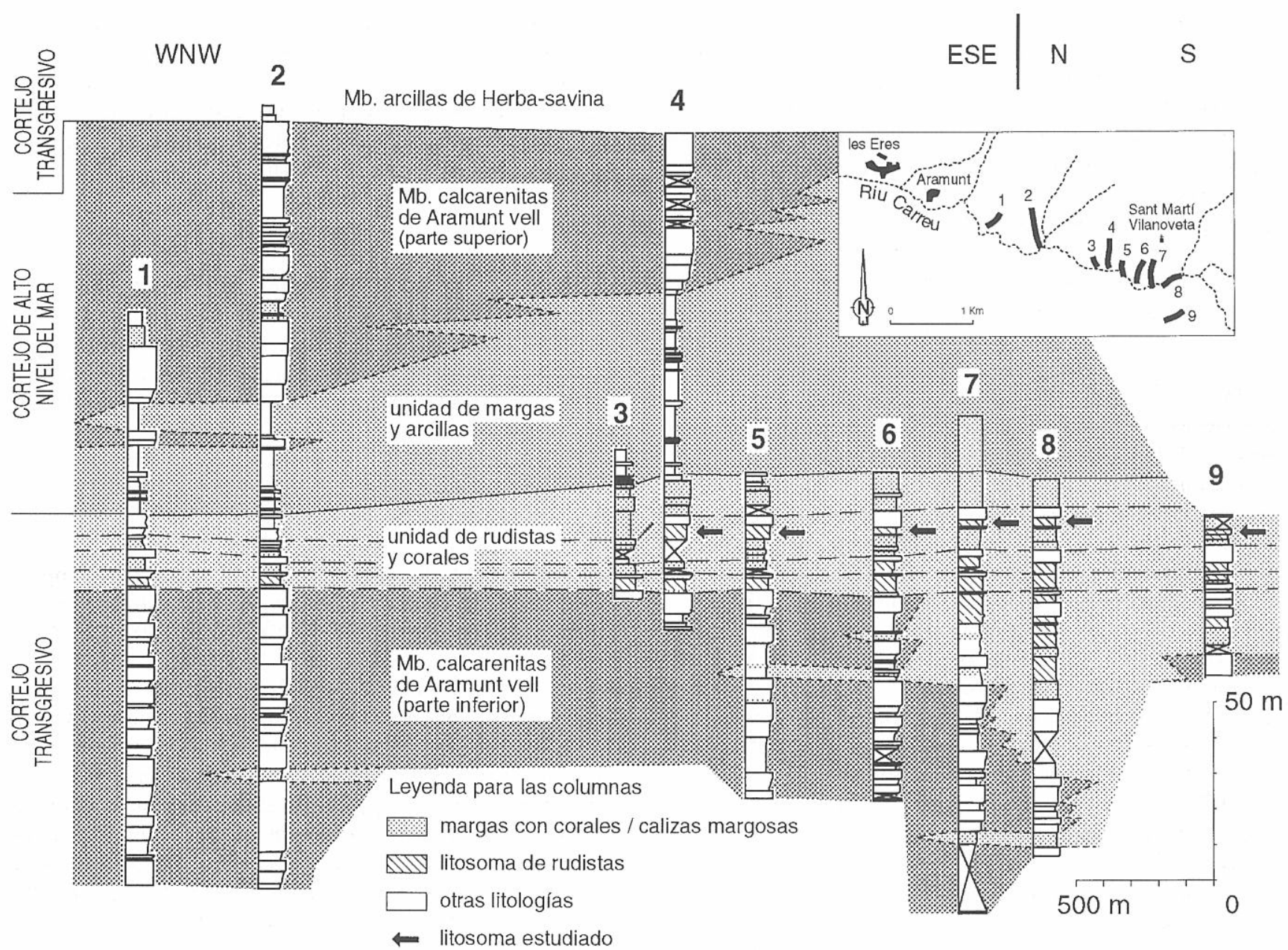

Figura 2. Sección estratigráfica de la parte superior de la plataforma de Sant Corneli mostrando las principales unidades litoestratigráficas y los cortejos sedimentarios interpretados.

margo-nodulosas que contienen fauna marina bentónica relativamente dispersa y restos vegetales muy pequeños. Estos depósitos pasan hacia techo a calcarenitas (grainstones) ocres, bioclásticas, con abundantes miliólidos y algunos granos de cuarzo, dispuestas en estratos gruesos. La dirección predominante de la estratificación oblicua es hacia el nordeste.

Estos depósitos han sido interpretados por Ross (1989: su "Facies A") como una sucesión de sets de dunas formados por corrientes de reflujo procedentes del sudoeste que transportaban una mezcla de material de origen marino y cierta cantidad de material de origen terrestre.

Lateralmente, hacia el este, estos materiales pasan a las facies de rudistas y corales que describimos seguidamente (Fig. 2).

\section{Unidad de rudistas y corales}

La parte inferior de esta unidad (series estratigráficas 7, 8 y 9 en la Fig. 2), lateralmente equivalente a las calcarenitas ocres de Aramunt vell inferior, está constituida por margas, calizas margo-nodulosas y calizas grises. Estas últimas pueden estar formadas por litosomas tabulares de rudistas (hipurítidos) cilíndrico-alargados, cubiertos, gene- ralmente, por depósitos bioclásticos (floatstones) con abundantes fragmentos de rudistas y corales.

La parte superior de la unidad (series estratigráficas 1-9 en la Fig. 2), está constituida por una serie de cuatro ciclos, cada vez más potentes, parecidos a las unidades de calizas grises antes mencionadas, que se extienden a lo largo de casi toda la plataforma.

El primero de estos ciclos es relativamente poco potente (menos de $6 \mathrm{~m}$ ), y remarcablemente consistente a lo largo de toda la exposición. Está constituido por un litosoma tabular de hipurítidos cilíndrico-alargados, no muy densamente empaquetados, cubierto por un depósito bioclástico de potencia variable.

Los dos ciclos siguientes $(6,5 \mathrm{~m}$ y $12 \mathrm{~m}$ de potencia máxima respectivamente) comienzan con un tramo de margas con abundantes corales y calizas margosas de aguas marinas abiertas, sobre el que yacen sendos litosomas tabulares de hipurítidos cubiertos por sedimentos bioclásticos (floatstones de rudistas que pasan hacia arriba a packstones/grainstones). Hacia el oeste, los litosomas de hipurítidos y los depósitos bioclásticos se acuñan, mientras que las margas con corales y calizas margosas persisten. Hemos interpretado, tanto el paso lateral (oeste-este), como la sucesión cíclica vertical en el este, de facies dominadas por corales a facies dominadas por 
rudistas, como una fase de somerización de la plataforma (Gili et al., en prensa) que termina con la llegada desde el nordeste de sedimentos bioclásticos procedentes del margen de la plataforma (Skelton et al., en prensa). La acumulación de margas con corales en la parte occidental de la unidad, sugiere la existencia de una ligera depresión en aquella zona, producto, quizás, de una tectónica sinsedimentaria. Esta hipótesis, no obstante, está aún en estudio.

El cuarto ciclo (17 m de potencia máxima) está constituido básicamente por un tramo inferior dominado por corales coloniales laminares, un tramo medio formado principalmente por corales laminares y domales, y un tramo superior constituido por una asociación mixta de Vaccinites (grandes hipurítidos) y corales. Hacia el oeste, los tramos se hacen muy margosos y aumenta notablemente la diversidad de organismos marinos bentónicos.

El aumento sucesivo de la potencia de estos cuatro ciclos y las diferencias en la sucesión de facies en los ciclos, implican una tendencia a aumentar la provisión de nuevo espacio acomodativo en el comienzo de cada nuevo ciclo.

Por encima de estos cuatro ciclos, las facies de rudistas y corales persisten sólo en la parte este de la sección, donde se observan diversos ciclos menores similares a los del último ciclo descrito, formando un resalte (sobre el que se asientan las ruinas del castillo de Vilanoveta). Sobre estos ciclos se desarrollan, a menudo, litosomas de rudistas formados casi exclusivamente por agregados de hipurítidos cilíndrico-alargados. Hemos interpretado que la sucesión faunística vertical observada en estos ciclos es consecuencia de la migración lateral de los diferentes ambientes sedimentarios presentes en la plataforma (Gili et al., en prensa) y no, como sugiere Ross (1989), el reflejo de una sucesión ecológica. Hacia el oeste, estas calizas se acuñan rápidamente dentro de las facies margosas que describimos a continuación.

\section{Unidad de margas y arcillas}

A la relativamente competente unidad de rudistas y corales le sucede una unidad más incompetente de margas, calizas margosas y arcillas. El desarrollo de capas más duras de calizas nodulosas, en los tramos margosos, sugiere la presencia de ciclos, especialmente en la parte inferior de la unidad. La fauna marina es diversa, especialmente en la parte inferior de la unidad, e incluye equínidos regulares e irregulares, corales solitarios, corales coloniales pequeños, braquiópodos, gasterópodos diversos, ostreidos (Pycnodonte (Phygraea) vesiculare (Lamarck)), y varias especies más de bivalvos epifaunales e infaunales, incluyendo cierta variedad de pequeños rudistas. Esta fauna y su matríz de grano fino implican condiciones marinas abiertas y tranquilas, en aguas relativamente profundas, y consecuentemente una nueva fase de máxima profundización.

Esta unidad pasa hacia el oeste a las calcarenitas de Aramunt vell superior.

\section{Miembro calcarenitas de Aramunt vell (parte superior)}

Esta parte del Miembro calcarenitas de Aramunt vell está constituida por ciclos sucesivos granocrecientes, como los que forman la parte inferior. Los ciclos progradan hacia el nordeste sobre las facies de margas y arcillas de la unidad infrayacente. Las facies margo-nodulosas de los tramos inferiores de los ciclos pueden contener bastantes macroforaminíferos bentónicos (Lacazina), restos vegetales muy pequeños y algunas gotas de ambar muy pequeñas. La migración lateral de esta unidad hacia el nordeste queda oscurecida por una combinación de fallas y erosión.

\section{Miembro arcillas de Herba-savina}

La parte superior del Miembro Aramunt vell se encuentra cubierta por un fondo endurecido, ferruginoso, colonizado por algunos rudistas, ostras y corales dispersos. Encima se encuentran las margas y calizas margosas del Miembro Herba-savina, que representan una profundización de la plataforma trascendental.

\section{HISTORIA DEPOSICIONAL}

Nuestra interpretación de la historia deposicional del Santoniense de la parte superior de la plataforma de Sant Corneli, en términos de estratigrafía secuencial, está también resumida en la Fig. 2. Como se deduce de la persistencia lateral de la estratificación tabular, la sedimentación tuvo lugar de una manera igualitaria y ubicua en toda la superficie superior de la plataforma, por lo que no hay geometrías estratales diagnósticas (onlap, downlap, etc.) visibles en el área estudiada. Sin embargo, el particular apilamiento de las facies en los ciclos, ocasionado por el cambio lateral del límite entre ellas, permite diferenciar dos cortejos sedimentarios: un cortejo transgresivo y un cortejo de alto nivel del mar, cuyo tránsito se identifica por el cambio en la evolución de las facies, de profundizante a somerizante.

El límite basal de las calcarenitas de Aramunt vell inferior con la unidad infrayacente (Miembro calizas con rudistas de Montagut (Gallemí et al, 1982)) no aflora en el área de estudio, y aunque aparece después de una importante falla al nordeste de la zona, aún no lo hemos estudiado con detalle.

En las calcarenitas de Aramunt vell inferior se observa una retrogradación concordante que permite interpretar este paquete sedimentario como un cortejo transgresivo. Por otra parte, los cuatro ciclos que constituyen el desarrollo principal de la unidad de rudistas y corales registran una sucesión de fases de profundización de amplitud creciente, juntamente con una disminución del aporte de sedimentos de Aramunt vell desde el sudoeste, que los identifica como un cortejo transgresivo. Por esto, provisionalmente, hemos reconocido la parte inferior del Miembro Aramunt vell (más las margas y calizas grises lateralmente equivalentes en el este) y los cuatro ciclos superiores de las facies de rudistas y corales, como dos fases sucesivas de un mismo cortejo trangresivo, el segundo con una expansión mayor de las facies marinas abiertas por toda la plataforma que el primero. El máximo de profundización marina está representado por la expansión de facies margosas de mar abierto sobre el cuarto ciclo de este último paquete sedimentario. Después, se registra una tendencia regresiva con el retorno desde el sudoeste de las calcarenitas de Aramunt vell superior. Así, conjuntamente, la unidad de margas y arcillas y la parte superior del Miembro calcarenitas de Aramunt vell forman un cortejo de alto nivel del mar (cf. Figura 4 de Van Wagoner et al., 1988). 
Es indudable que el Miembro Herba-savina representa una fase importante de profundización, pero no hemos encontrado ninguna evidencia de exposición subaérea en la superficie superior de la plataforma. Por el contrario, el fondo endurecido simplemente sugiere un hiato deposicional, tal como el que podría haber acompañado a una profundización rápida que superara la velocidad de producción de carbonato en el área. Podría, pues, tratarse de una drowning unconformity sensu Schlager (1991), pero no tenemos aún suficientes datos para probar esta hipótesis.

Finalmente, aunque la parte de la secuencia sedimentaria estudiada no haya sido datada, dentro del Santoniense, con precisión suficiente para hacer comparaciones fidedignas con las curvas eustáticas postuladas, hemos de señalar que existe cierta similitud con el ciclo eustático del Santoniense UZA 3.3 de Haq et al. (1988). No obstante, necesitamos ciertamente análisis tectonoestratigráficos más precisos y correlaciones bioestratigráficas más refinadas para poder distinguir con plena confianza la influencia relativa de la eustasia, la tectónica y la sedimentación.

\section{PALEOECOLOGÍA}

En este apartado describimos brevemente e interpretamos la formación de un extenso litosoma de rudistas contenido en una capa de la sección (parte superior del tercer ciclo de los cuatro que forman el desarrollo principal de la unidad de rudistas y corales) (ver Skelton et al., en prensa, para una exposición más amplia del tema). Su elección se debió a su buen afloramiento a lo largo de 1,25 Km de oeste a este de la sección y varios centenares de metros hacia el S (Fig. 2). Su estudio se ha basado en las observaciones realizadas en 22 series estratigráficas muy detalladas y correlacionables lateralmente.

\section{DESCRIPCIÓN}

\section{Geometría del litosoma y contexto}

El litosoma de rudistas (hipurítidos) estudiado tiene forma tabular, una potencia máxima de $6 \mathrm{~m}$, en el oeste y sur (aunque generalmente no pasa de 1,5 a 2 m) y una extensión de $1,25 \mathrm{Km}^{2}$. Hacia el norte se encuentra fallado y cubierto por sedimentos más modernos, hacia el este y sur se pierde por erosión, y hacia el oeste es reemplazado por una cuña de calizas margo-nodulosas con corales. La mayor parte del litosoma yace sobre una delgada capa de packstones bioclásticos de potencia remarcablemente constante (máximo $80 \mathrm{~cm}$, pero en general menos de $50 \mathrm{~cm}$ ), que también se acuña hacia el oeste. Los bioclastos están mal clasificados y son predominantemente fragmentos angulares de rudistas, equinidos $\mathrm{y}$ corales, algas rojas incrustantes y foraminíferos bentónicos (Lámina I, a). Debajo se encuentra una capa de calizas margo-nodulosas y margas, con abundante corales coloniales (planos a domales) y chaetétidos. Hacia el techo de esta capa son comunes los rudistas grandes solitarios (Vaccinites).

El litosoma de hipurítidos se encuentra cubierto por una capa, también de potencia muy constante (máximo 4,4 m) y acuñación hacia el oeste, de floatstones de rudistas (en una matriz wackestone/packstone bioclástica) que pasan hacia arriba a packstones o raramente grainstones, bioclásticos, Los bioclastos más abundantes son fragmentos subangulares, mal clasificados de rudistas (radiolítidos, hipurítidos), gasterópodos, equínidos y corales, algas rojas incrustantes y foraminíferos bentónicos (Lámina I, b). La asociación de litosomas de hipurítidos tabulares con capas bioclásticas suprayacentes se ha observado también en otras partes, tanto en la sección de Sant Martí de Vilanoveta como en les Collades de Basturs (Gili, 1984). Localmente se observa una estratificación mal definida, que en algunos casos presenta una ligera inclinación hacia el oeste/sudoeste.

\section{Composición faunística y desarrollo del litosoma}

Los litosomas de rudistas, tanto en esta sección como en les Collades de Basturs, son notablemente pauciespecíficos. En esta última sección los agregados están formados por sólo de una a tres especies de hipurítidos (Gili, 1992). En Sant Martí de Vilanoveta, muy a menudo, los agregados están dominados por una sola especie. En el litosoma de hipurítidos que estamos describiendo, la gran mayoría de individuos son Hippurites socialis Douvillé. Otros hipurítidos elevadores presentes en el litosoma son $H$. praecesor Douvillé, y, mucho más raramente $H$. sublaevis Matheron y otras especies de Hippurites, Hippuritella y Vaccinites.

La fauna asociada es escasa y constituida principalmente por otros rudistas (Plagioptychus paradoxus Matheron y diversos radiolítidos), junto con algunos corales planos o ligeramente domales y chaetétidos.

En la base del litosoma los hipurítidos (de una longitud máxima de $15 \mathrm{~cm}$ ) están agrupados en pequeños manojos verticales (bouquet sensu Philip, 1972) (Lámina I, c). En los agregados principales, por el contrario, las conchas verticales o ligeramente inclinadas están instaladas en los flancos de otras conchas o bien en conchas caidas o en pequeños fragmentos de conchas Estos hipurítidos pueden alcanzar $40 \mathrm{~cm}$ de largo, aunque los individuos de más de $30 \mathrm{~cm}$ son raros. En estos agregados, generalmente, los hipurítidos que se conservan más o menos verticales forman grupos compactos de pocos individuos (comúnmente de dos a cuatro), separados por una matriz bioclástica (Lámina I, d).

\section{Tafonomía}

No obstante y a pesar de lo expuesto en el párrafo anterior, la mayoría de las conchas del litosoma de hipurítidos, por encima del tramo basal de bouquets, están orientadas paralelamente o subparalelamente a la estratificación, generalmente densamente empaquetadas, y con la comisura apuntando hacia el cuadrante sudoeste (Lámina I, e). La disposición de las tábulas de estos hipurítidos, perpendiculares a las paredes de la concha, implica que éstas crecieron verticalmente (Skelton y Gili, 1991; Gili y Skelton, 1994), y que por lo tanto esta orientación no corresponde a la de su posición de vida.

\section{Microfacies}

La matriz del litosoma de hipurítidos está constituida principalmente por fragmentos grandes de hipurítidos muy 


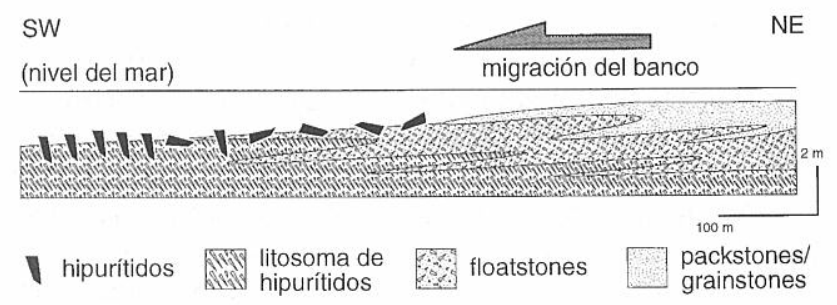

Figura 3. Modelo deposicional del litosoma de hipurítidos. La escala horizontal es aproximada.

bioperforados, angulares y muy mal clasificados, y por fragmentos pequeños, de tamaño arena a limo, también de hipurítidos, junto con una micrita oscura (Lámina I, f).

\section{INTERPRETACIÓN}

La uniformidad de la capa bioclástica infrayacente en un área tan extensa, sugiere que el litosoma de hipurítidos se desarrolló en una superficie más o menos llana. La forma tabular del mismo litosoma y de los depósitos bioclásticos suprayacentes, implica un espacio acomodativo uniforme, y por lo tanto acumulación en lo que probablemente fué una superficie más o menos horizontal en la parte superior de la plataforma. El cambio de facies asociado con el acuñamiento del litosoma hacia el oeste, puede que esté relacionado con un cambio en la profundidad del agua, o simplemente con una variación lateral en la actividad de las corrientes, o con cualquier otro factor que implique una conexión mayor con el mar abierto.

La relativa uniformidad de la potencia de la capa bioclástica que cubre el litosoma de hipurítidos y la evidencia, local, de la estratificación oblicua, sugiere la propagación lateral hacia el oeste/sudoeste de las facies bioclásticas sobre la superficie superior del litosoma (Fig. 3).

Siendo organismos aclonales, cada hipurítido en su etapa larval se fijaba individualmente sobre una superficie dura, tal como un fragmento de concha u otra concha (Skelton y Gili, 1991; Gili y Skelton, 1994). La formación de manojos pequeños, aislados, en la base del litosoma, y de agregados más grandes por encima de éstos, posiblemente refleja, respectivamente, una menor o mayor disponibilidad de substratos para fijarse.

La naturaleza pauciespecífica de los agregados de hipurítidos y la práctica ausencia de fauna asociada, puede ser debida a que las condiciones ambientales reinantes (por ejemplo, circulación marina restringida) en las zonas someras de la plataforma, en las que los hipurítidos crecían, fueran desfavorables para otros organismos (Gili et al., en prensa).

En los agregados, las conchas de los hipurítidos estaban sostenidas en parte por el sedimento que se depositaba a su alrededor a medida que iban creciendo, y en parte por sus vecinos. Ocasionalmente, los agregados eran afectados por corrientes (probablemente inducidas por tempestades) que removían el sedimento, provocando la caída (en "dominó") de las conchas. El predominio de la orientación de los hipurítidos caídos hacia el cuadrante sudoeste, implica que estas corrientes provenían predominantemente del nordeste.
Los sedimentos bioclásticos que se encuentran por debajo y por encima del litosoma tienen características de mar notablemente más abierto que la matriz del litosoma. Contienen una diversidad mayor de bioclastos que ésta última, y tienen texturas que indican una actividad mayor de las corrientes. Este hecho y la evidencia de la propagación lateral de estos sedimentos (discutida anteriormente), indica que este material fue transportado desde una zona marina más abierta (margen de la plataforma) situada en el nordeste.

La fracción bioclástica de la matriz del litosoma parece ser mayormente producto de la biodegradación local de conchas de los hipurítidos, acumulado in situ entre los rudistas, en condiciones energéticas normalmente tranquilas, ocasionalmente perturbadas por corrientes de tempestad. La intensa bioerosión presente en este ambiente, sugiere condiciones moderadamente ricas en nutrientes (mesotróficas) (Hallock, 1988), como las reconocidas en les Collades de Basturs (Gili, 1992), y La Cadière en el sudeste de Francia (Grosheny y Philip, 1989).

\section{CONCLUSIONES}

De todo lo expuesto anteriormente sobre los agregados de hipurítidos, hemos extraído las conclusiones siguientes, que son aplicables a la mayoría de los litosomas de hipurítidos de la sección.

1. Los rudistas elevadores crecían en manojos y agregados laxos, enclavados en, y sostenidos por, el sedimento que se acumulaba a su alrededor, sobresaliendo sólo una pequeña parte de la concha por encima de la superficie del substrato. Para este tipo de crecimiento, Gili et al. (en prensa) han propuesto el término constratal, en oposición al crecimiento superstratal, término también propuesto por Gili et al. (en prensa), en el cual las conchas o esqueletos estarían en gran parte expuestos, sobresaliendo ampliamente del substrato. No hay ninguna evidencia de que los agregados de hipurítidos formaran una estructura orgánica, elevada, capaz de autosostenerse. De hecho, ocasionalmente, la remoción de sedimento intersticial, ocasionada probablemente por corrientes de tempestad, provocaba el colapso y apilamiento de las conchas. Así, la estructura del litosoma está constituida por rudistas horizontales, caídos, y rudistas verticales o inclinados en posición de vida.

2. La mayor parte del sedimento en el que crecían los agregados de hipurítidos, provenía de la biodegradación local de conchas preexistentes. Muy poco material de fuera era introducido en ellos, por lo que, en rigor, no se los puede denominar bafflestones. Finalmente, sin embargo, fueron progresivamente cubiertos por sedimentos bioclásticos transportados desde áreas marinas más abiertas (del margen de la plataforma en el nordeste) (Fig. 3).

3. Los agregados de hipurítidos no tenían relieve. Por el contrario, ocupaban el nivel del fondo de amplias áreas de la plataforma, detrás de la zona marginal, más barrida por las corrientes, que servía de área fuente a los depósitos bioclásticos. 
En resumen, pues, los hipurítidos crecieron como habitantes de los sedimentos del fondo de la superficie superior de la plataforma, en aguas generalmente quietas, donde ocasionalmente eran perturbados por tormentas. Allí generaron los extensos litosomas tabulares que constituyen una parte muy importante de las formaciones de rudistas de Sant Martí de Vilanoveta.

Por último, queremos señalar que en vista del crecimiento conestratal (sin desarrollo de estructura rígida), y de la ausencia de relieve original en los agregados, consideramos que el término "arrecifal", aplicado frecuentemente en la literatura, a estos litosomas y a los de les Collades de Basturs, es totalmente inapropiado.

\section{AGRADECIMIENTOS}

Este estudio ha estado subvencionado por la DGICYT, proyecto de investigación No. PS90-0171. Los autores agradecen también la ayuda financiera del Ministerio de Educación y Ciencia y del British Council (Acción integrada Hispano-Británica 1993/94 No. 153B/190 y 1994/95 No. HB93-106) que ha facilitado la colaboración entre el equipo español y PWS. El análisis bioestratigráfico ha contado con la inestimable colaboración de W. J. Kennedy (Universidad de Oxford, G. B.) y E. A. M. Koutsoukus (Petrobras, Brasil). A Alfonso Meléndez y a un revisor anónimo expresamos nuestro reconocimiento por los útiles comentarios realizados al texto original.

\section{BIBLIOGRAFÍA}

Gallemí, J., Martínez, R. y Pons, J. M. 1982. Unidades del Cretácico superior en los alrededores de Sant Corneli (Provincia de Lleida). Cuadernos de Geología Ibérica, 8, 935-948.

Gallemí, J., Martínez, R. and Pons, J. M. 1983. Coniacian-Masstrichtian of the Tremp Area (South Central Pyrenees). Newsleter Stratigraphy, 12, 1-17.

Gili, E. 1984. Interaccions sedimentològiques i biològiques a les formacions calcàries de rudistes (Bivalvia) de Les Collades de Basturs (Cretaci superior, zona sudpirinenca central). Resum de Tesi doctoral, Universitat Autònoma de Barcelona, Bellaterra. 1-42.

Gili, E. 1992. Palaeoecological significance of rudist constructions: a case study from les Collades de Basturs (Upper Cretaceous, South Central Pyrenees). Geologica Romana, 28, 319-325.
Gili, E. 1993. Facies and geometry of les Collades de Basturs carbonate platform, Upper Cretaceous, South Central Pyrenees. In: Cretaceous Carbonate Platforms (Eds. J. A. Toni Simo, R. W. Scott y J. P. Masse). American Association of Petroleum Geologists, Memoir 56, Tulsa, 343-352.

Gili, E. i Skelton, P. W. 1994. Classificació paleoecològica de les formes dels rudistes - una eina per a l'anàlisi paleoambiental. Butlletí de la Institució Catalana d'Història Natural, 61, 97-116.

Gili, E., López, G., Obrador, A., Skelton, P. W. y Vicens, E. 1994. Observaciones sobre la posición estratigráfica de las formaciones de rudistas de Sant Corneli (Cuenca Cretácica Surpirenaica Central). Geogaceta, 15, 34-36.

Gili, E., Skelton, P. W., Vicens, E. y Obrador, A. (en prensa). Corals to rudists: an environmentally induced assemblage sequence. In: Palaeoenvironmental models for the benthic associations of Tethyan Cretaceous carbonate platforms. (Eds. J. Philip y P. W. Skelton), Palaeogeography, Palaeoclimatology, Palaeoecology.

Grosheny, D. and Philip, J. 1989. Dynamique biosédimentaire de bancs à rudistes dans un environnement pérideltaïque: la formation de La Cadière d'Azur (Santonien, SE France). Bullétin du Société géologique de France, 8 (5), 1253-1269.

Hallock, P. 1988. The role of nutrient availability in bioerosion: consequences to carbonate buildups. Palaeogeography, Palaeoclimatology, Palaeoecology, 63, 275-291.

Hancock, J. M. 1991. Ammonite scales for the Cretaceous system. Cretaceous Research, 12, 259-291.

Haq, B. U., Hardenbol, J. and Vail, P. R. 1988. Mesozoic and Cenozoic Chronostratigraphy and cicles of sea-level change. In: Sea-level changes - An integrated approach, (Eds.: C. K. Wilgus, B. S. Hastings, C. G. Kendall, H. Posamentier, C. A. Ross y J. C. Van Wagoner), Society of Economic Paleontologists and Mineralogists, Special Publication, 42, 71-108.

Kennedy, W. J. 1987. Ammonites from the type Santonian and adjacent parts of northern Aquitaine, western France. Palaeonto$\log y, 30$ (4), 765-782.

López, G. 1992. Paleontología y Bioestratigrafía de los inocerámidos (Bivalvia) del Cretácico superior de la Cuenca NavarroCántabra y de la Plataforma Norcastellana. Parte IV: Estudio sistemático del subgénero Cordiceramus Seitz y Bioestratigrafía. Boletín Geológico y Minero, 103 (5), 837-892.

Martínez, R. 1982. Ammonoideos cretácicos del Prepirineo de la provincia de Lleida. Publicaciones de Geología, Universidad Autónoma de Barcelona, 17, 1-198.

Muñoz, J. A. 1992. Evolution of a continental collision belt; ECORSPyrenees crustal balanced cross-section. In: Thrust Tectonics (Ed. K.R. McClay) Chapman y Hall, London, 235-246.

\section{Lámina I}

a) Packstone bioclástico de la capa que se encuentra debajo del litosoma de hipurítidos. Fragmento de radiolítido (concha celuloprismática) en el centro de la mitad inferior de la fotografía, debajo de un foraminífero bentónico. La barra equivale a 1 $\mathrm{mm}$.

b) Packstone bioclástico de la capa que cubre el litosoma de hipurítidos. La barra equivale a $1 \mathrm{~mm}$.

c) Manojos verticales (bouquets) de hipurítidos en la base del litosoma de rudistas. El martillo mide $33 \mathrm{~cm}$ de largo.

d) Agregado de hipurítidos. Los rudistas son Hippurites socialis Douvillé. La barra equivale a $10 \mathrm{~cm}$.

e) Aspecto general del litosoma de hipurítidos. Las conchas se encuentran fuertemente inclinadas hacia la izquierda, en el sentido de las corrientes dominantes. Hemos interpretado que esta orientación es deposicional y que no corresponde a su posición de vida. La tapa del objetivo mide $5,5 \mathrm{~cm}$

f) Microfacies de la matriz del litosoma de hipurítidos. En los márgenes inferior e izquierdo se observan fragmentos grandes de hipurítidos con bioperforaciones. La barra equivale a $1 \mathrm{~mm}$. 
Lámina I
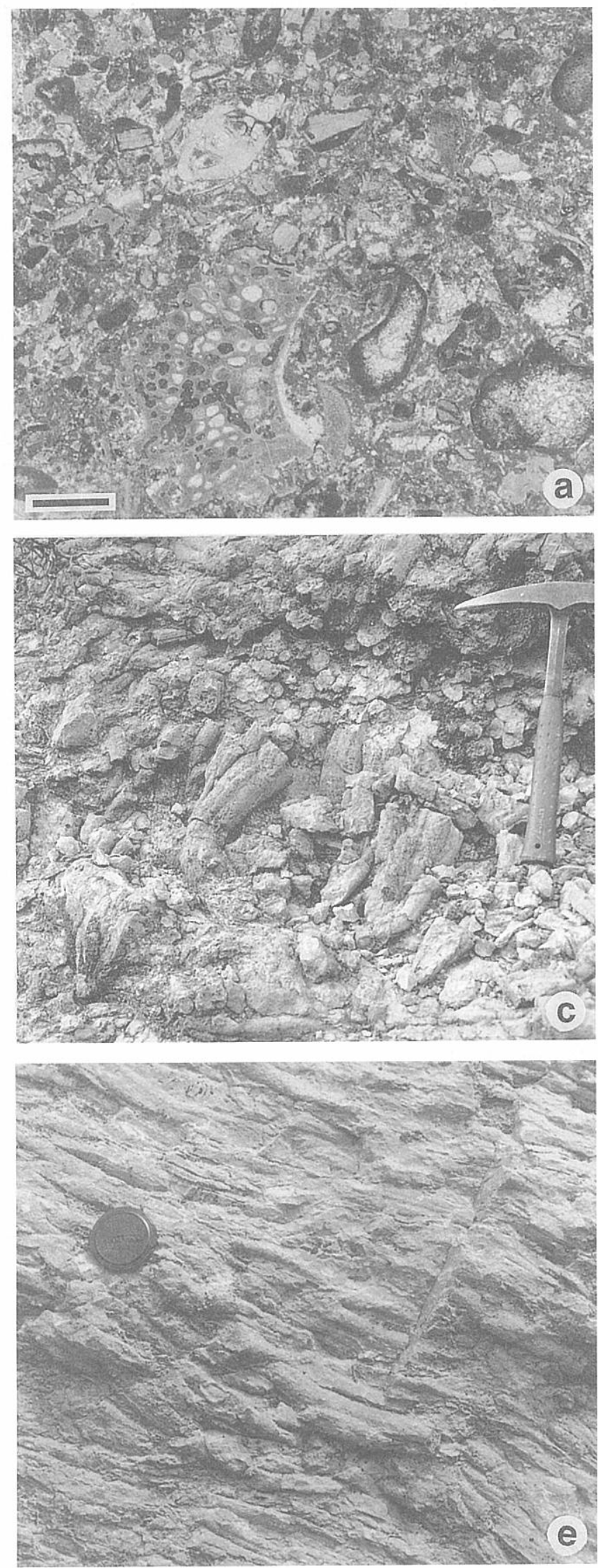

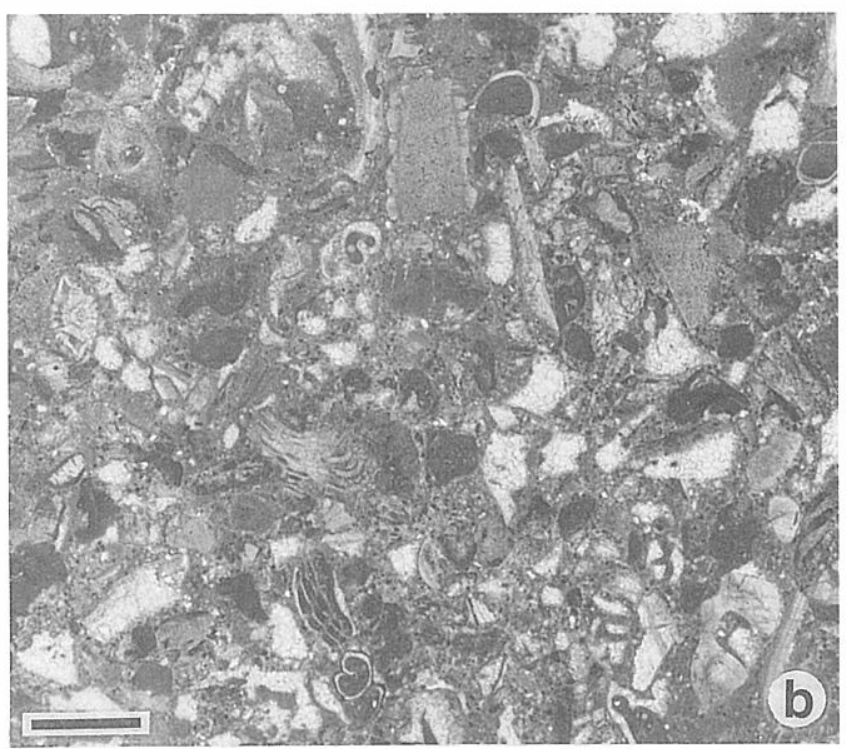

3.71 .

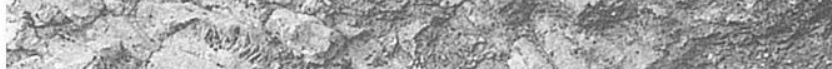

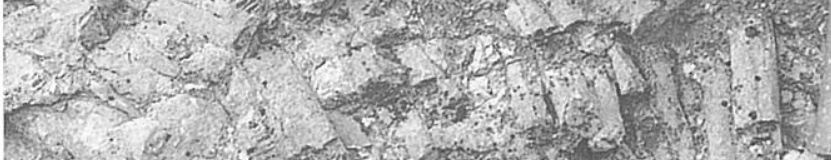

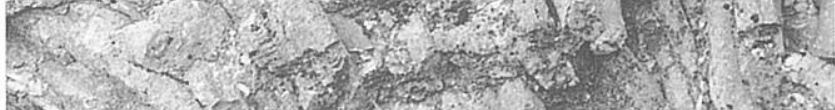

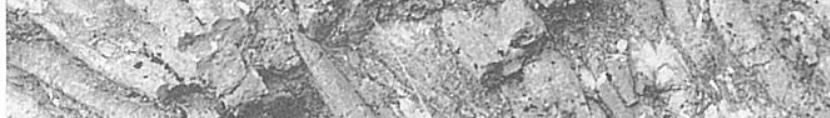

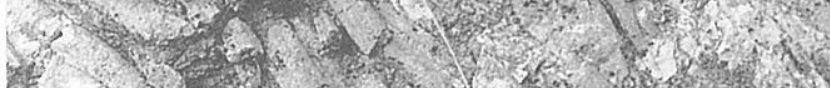

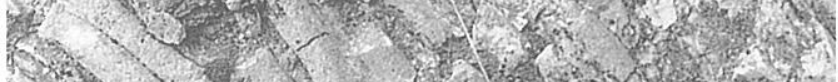

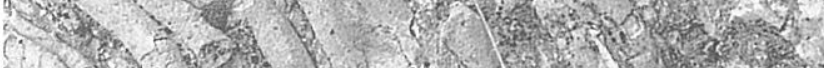

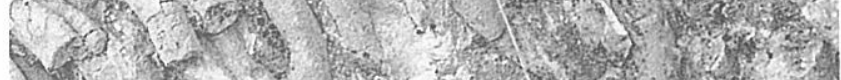

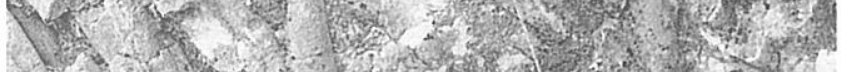

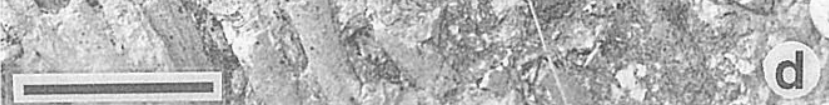

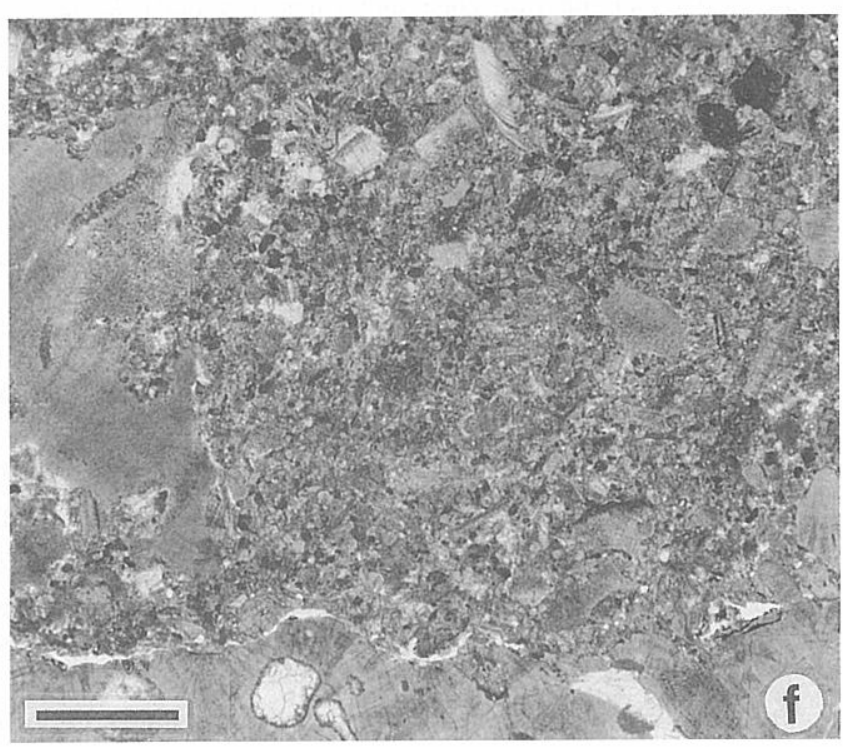


Philip, J. 1972. Paléoécologie des formations à rudistes du Crétacé supérieur -l'exemple du Sud-Est de la France. Palaeogeography, Palaeoclimatology, Palaeoecology, 12, 205-222.

Rosell, J. 1967. Estudio geológico del sector del Prepirineo comprendido entre los ríos Segre y Noguera Ribagorzana (Provincia de Lérida). Pirineos, 75-78, 1-225.

Ross, D. J. 1989. Facies analysis and diagenesis of Tethyan rudist reefs complexes. Ph. D. Thesis, University of Wales, Cardiff, 206 pp.

Santamaría, R. 1992. Los ammonoideos del Cenomaniense superior al Santoniense de la Plataforma Nord-Castellana y la Cuenca Navarro-Cántabra Parte I. Bioestratigrafía y sistemática: Phylloceratina, Ammonitina (Desmocerataceae y Hoplitaceae) y Ancyloceratina. Treballs del Museu de Geologia de Barcelo$n a, 2,171-268$.

Schlager, W. 1991. Depositional bias and environmental changeimportant factors in sequence stratigraphy. Sedimentary Geology, 70, 109-130.

Seguret, M. 1972. Étude tectonique des nappes et séries décollées de la partie centrale du versant sud des Pyrénées. Publications de l'Université de Sciences et Techniques de Languedoc série Geologie Structurale, 2. Montpellier, 155 pp.

Seitz, O. 1961. Die Inoceramen des Santon von Nordwestdeutschland. I Teil. Platyceramus, Cladoceramus und Cordiceramus. Beihefte zum Geologischen Jahrbuch, 46, 3-186.

Simó, J. A. 1993. Cretaceous Carbonate Platforms and Stratigraphic Sequences, South-Central Pyrenees, Spain. In: Cretaceous Carbonate Platforms (Eds. J. A. Toni Simo, R.W. Scott y J.P. Masse). American Association of Petroleum Geologists, Memoir 56, 325-342.
Skelton, P. W. and Gili, E. 1991 (en prensa). Palaeoecological classification of rudist morphotypes. Proceeding $I^{s t}$ Conference on Rudist (Beograd, 1988). Serbian Geological Society. Special Publication 2, 265-287.

Skelton, P. W., Gili, E., Vicens, E. and Obrador, A. (en prensa). The growth fabric of gregarious rudist elevators (hippuritids) in the Santonian carbonate platforms of the Tremp area, Central southern Pyrenees. In: Palaeoenvironmental models for the benthic associations of Tethyan Cretaceous carbonate platforms. (Eds. J. Philip y P. W. Skelton), Palaeogeography, Palaeoclimatology, Palaeoecology.

Tröger, K. A. 1987. Problems of Upper Cretaceous Inoceramid Biostratigraphy and Paleobiogeography in Europe and Western Asia. In: Cretaceous of the Western Tethys. Proceedings $3 r$ international Cretaceous Symposium, Tübingen 1987 (Ed. J. Wiedmann). E. Schweizerbart'sche Verlagsbuchhandlung. Stuttgart, 911-930.

Van Wagoner, J. C., Posamentier, H. W., Mitchum, R. M., Vail, P. R., Sarg, J. F., Loutit, T. S. and Hardenbol, J. 1988. An overview of the fundamentals of sequence stratigraphy and key definitions. In: Sea-level changes - An integrated approach, (Eds.: C. K. Wilgus, B. S. Hastings, C. G. Kendall, H. Posamentier, C. A. Ross y J. C. Van Wagoner), Society of Economic Paleontologists and Mineralogists, Special Publication, 42, 39-45.

Manuscrito recibido: 26 de enero, 1995 Manuscrito aceptado: 16 de julio, 1995 